\title{
Nanoscale Organization of the MEC-4 DEG/ENaC Sensory Mechanotransduction Channel in Caenorhabditis elegans Touch Receptor Neurons
}

\author{
Juan G. Cueva, Atticus Mulholland, and Miriam B. Goodman \\ Department of Molecular and Cellular Physiology, Stanford University School of Medicine, Stanford, California 94305
}

\begin{abstract}
Hearing, touch and proprioception are thought to involve direct activation of mechano-electrical transduction (MeT) channels. In Caenorhabditis elegans touch receptor neurons (TRNs), such channels contain two pore-forming subunits (MEC-4 and MEC-10) and two auxiliary subunits (MEC-2 and MEC-6). MEC-4 and MEC-10 belong to a large superfamily of ion channel proteins (DEG/ENaCs) that form nonvoltage-gated, amiloride-sensitive $\mathrm{Na}^{+}$channels. In TRNs, unique 15-protofilament microtubules and an electron-dense extracellular matrix have been proposed to serve as gating tethers critical for MeT channel activation. We combined high-pressure freezing and serial-section immunoelectron microscopy to determine the position of $\mathrm{MeT}$ channels relative to putative gating tethers. MeT channels were visualized using antibodies against MEC-4 and MEC-2. This nanometer-resolution view of a sensory MeT channel establishes structural constraints on the mechanics of channel gating. We show here that MEC-2 and MEC-5 collagen, a putative extracellular tether, occupy overlapping but distinct domains in TRN neurites. Although channels decorate all sides of TRN neurites; they are not associated with the distal endpoints of 15-protofilament microtubules hypothesized to be gating tethers. These specialized microtubules, which are unique to TRNs, assemble into a cross-linked bundle connected by a network of kinked filaments to the neurite membrane. We speculate that the microtubule bundle converts external point loads into membrane stretch which, in turn, facilitates MeT channel activation.
\end{abstract}

Key words: somatosensory; mechanosensory; cytoskeleton; $\mathrm{ENaC}$ (epithelial sodium channels); transduction; C. elegans

\section{Introduction}

The skin is endowed with a rich array of touch receptor cells, each of which is thought to express ion channels activated by force or displacement. Functionally analogous channels underlie our sense of hearing. Because they activate with submillisecond latencies (Corey and Hudspeth, 1979; Walker et al., 2000; O'Hagan et al., 2005), these so-called mechano-electrical transduction or MeT channels are believed to both sense force and carry receptor currents. Understanding the mechanics of channel activation remains a challenge that will require a combination of approaches, including quantitative ultrastructural analysis.

The touch receptor neurons (TRNs) of the nematode Caenorhabditis elegans are ideally suited for this analysis. They are readily identifiable in living animals and in electron micrographs of animal cross-sections and mediate avoidance of low-intensity touches (Chalfie and Sulston, 1981; Chalfie et al., 1985). Genetically encoded $\mathrm{Ca}^{2+}$ sensors (Suzuki et al., 2003) and whole-cell

Received Sept. 12, 2007; revised 0ct. 31, 2007; accepted Nov. 1, 2007.

This work was supported by National Institutes of Health Grant NS47715 (M.B.G.), by fellowships from the McKnight, A. P. Sloan, and Donald B. and Delia E. Baxter Foundations (M.B.G.), and by an American Psychological Association Diversity Program in Neuroscience Postdoctoral fellowship (J.G.C). We thank K. McDonald for a donation of embedded samples of wild-type (N2) animals at the early stages of this research, J. Mulholland and J. Perrino for expert technical assistance, N. Dompe for animating three-dimensional reconstructions, D. Ramot for simulating a random distribution of label in a realistic model TRN, and D. Lenzi, R. S. Lewis, and A. Ricci for comments.

Correspondence should be addressed to Dr. Miriam B. Goodman, B-111 Beckman Center, 279 Campus Drive, Stanford, CA 94305. E-mail: mbgoodman@stanford.edu.

D0I:10.1523/JNEUROSCI.4179-07.2007

Copyright $\odot 2007$ Society for Neuroscience 0270-6474/07/2714089-10\$15.00/0 patch clamp recording (O'Hagan et al., 2005) have been used to measure TRN responses in vivo. Four membrane proteins, including two pore-forming subunits (MEC-4 and MEC-10) and two accessory proteins (MEC-2 and MEC-6) are known to be subunits of the native MeT channel (O'Hagan et al., 2005). In heterologous cells, they coimmunoprecipitate one another and form nonvoltage-gated, amiloride-sensitive $\mathrm{Na}^{+}$channels (Chelur et al., 2002; Goodman et al., 2002). UNC-24, a membrane protein related to MEC-2 (Barnes et al., 1996) may also contribute to the complex (Zhang et al., 2004). Saturating mechanical stimuli activate 14-25 MeT channels in PLM neurons (O'Hagan et al., 2005), a pair of TRNs which innervate the posterior half of the body surface. Because a single PLM neuron is sufficient to confer touch sensitivity in its receptive field (Chalfie and Sulston, 1981; Chalfie et al., 1985), this figure suggests a remarkable conclusion: Activation of $<25 \mathrm{MeT}$ channels can initiate a behavioral response.

Genetic evidence suggests that proper activation of MeT channels relies on 15-protofilament (15pf) microtubules and an electron-dense, lectin-binding extracellular matrix (ECM) (Ernstrom and Chalfie, 2002; Goodman and Schwarz, 2003). A widely discussed model based on analysis of interactions between the genes encoding the MeT channel (mec-4, mec-10, mec-2, mec-6) and those encoding TRN-enriched tubulins (mec-7 and mec-12) and secreted ECM proteins ( $m e c-1$, mec-5, and mec-9) posits that MeT channels localize to junctions between the distal endpoints of $15 \mathrm{pf}$ microtubules and the plasma membrane and form the 
core of a trans-cellular mechanotransduction complex in which channels open in response to differential displacement of microtubules and the ECM (Huang and Chalfie, 1994; Huang et al., 1995; Gu et al., 1996). The structural details of the proposed in vivo interactions remain unknown.

In the present study we used serial-section immunoelectron microscopy (SS-IEM) of adult C. elegans hermaphrodites to examine, in three dimensions and with nanometer resolution, the organization of $\mathrm{MeT}$ channels and their position relative to the TRN cytoskeleton and ECM and interpret these results in the context of previous genetic and physiological analysis of these cells. To minimize fixation-induced osmotic shock and maximize preservation of ultrastructure and protein antigens, we fixed animals using high-pressure freezing and freeze substitution (McDonald and Auer, 2006). We analyzed the longitudinal and circumferential position of MeT channels (visualized with antiMEC-2 and anti-MEC-4 antibodies) with respect to $15 \mathrm{pf}$ microtubules, the electron-dense ECM, and the secreted ECM protein, MEC-5. We report that MeT channels and MEC-5 occupy overlapping, but distinct immunofluorescence domains in TRN neurites and that, although MeT channels decorate all sides of TRN neurites, they do not localize to junctions between the plasma membrane and distal endpoints of $15 \mathrm{pf}$ microtubules. These microtubules assemble into a cross-linked bundle connected to the plasma membrane by a network of kinked filaments. These structural data constrain functional models that can be tested in future physiological, genetic and anatomical studies of this mechanoreceptor neuron.

\section{Materials and Methods}

C. elegans strains. The culture and genetics of wild-type C. elegans Bristol (N2) and its mutants followed Brenner (1974). Wild-type worms were N2 animals provided by the Caenorhabditis Genetics Center, which is funded by the NIH National Center for Research Resources (NCRR), or TU2769, a transgenic animal that expresses GFP under the control of the mec-17 promoter (O'Hagan et al., 2005). TU2769 animals have no visible anatomical phenotypes or obvious defects in locomotion and exhibit nearly wild-type touch sensitivity (O'Hagan et al., 2005). Null mutants, mec-2(u37), mec-4(u253), and mec-5(u2020) were provided by Martin Chalfie (Columbia University, NY, New York) and crossed into TU2769 for immunofluorescence studies.

Antibodies. Polyclonal antibodies against MEC-2 (amino acids 19118), MEC-4 (amino acids 223-322), and MEC-5 (amino acids 103-203) were generated by genetic immunization in mice at the antibody core of the University of Texas Southwestern (Tang et al., 1992; Chambers and Johnston, 2003) and are available from Abcam (Cambridge, MA). In all experiments, the MEC-2, MEC-4, and MEC-5 antibodies were preadsorbed against total protein homogenate from mec-2(u37), mec-4(u253), and mec-5(u2020) null alleles. Preadsorption reduces background by decreasing the concentration of antibodies that may recognize antigenic sequences of closely related proteins while minimizing the loss of highaffinity antibodies. A rabbit polyclonal antibody directed against MEC-2 was used for double-labeling experiments (Zhang et al., 2004).

Indirect immunofluorescence. Adult, wild type and mutant nematodes were prepared for immunofluorescence in one of two fixation solutions: $4 \%$ paraformaldehyde in $1 \times \mathrm{PBS}, \mathrm{pH} 7.2$ or Ruvkun-Finney fixative $(160$ mм KCl, 40 mм NaCl, 10 mм Na 2 EGTA, 10 mm spermidine $\mathrm{HCl}, 30 \mathrm{~mm}$ NaPIPES, 25\% methanol, and 2\% paraformaldehyde). Fixed nematodes were subsequently permeabilized following a modified peroxide tube fixation protocol. Briefly, fixed nematodes were washed twice in TrisTriton solution (TTS; $100 \mathrm{~mm}$ Tris, $\mathrm{pH} 7.4,1 \%$ Triton X-100, $1 \mathrm{~mm}$ EDTA), incubated $4 \mathrm{~h}$ in TTS plus $1 \% \beta$-mercaptoethanol at $37^{\circ} \mathrm{C}$ with mild agitation. The animals were next washed with Borate Buffer solution $\left(\mathrm{BO}_{3} \mathrm{~B} ; 25 \mathrm{~mm} \mathrm{H}_{3} \mathrm{BO}_{3}, 0.01 \%\right.$ Triton X-100, $\mathrm{pH}$ 9.5), incubated for $15 \mathrm{~min}$ in $\mathrm{BO}_{3} \mathrm{~B}$ plus $10 \mathrm{~mm}$ dithiotreitol at $37^{\circ} \mathrm{C}$, washed again with $\mathrm{BO}_{3} \mathrm{~B}$, and incubated $15 \mathrm{~min}$ in $\mathrm{BO}_{3} \mathrm{~B}$ plus $0.3 \% \mathrm{H}_{2} \mathrm{O}_{2}$ at room temper- ature. Permeabilized nematodes were stored in Antibody Buffer (AbB; $1 \times$ PBS, $0.5 \%$ Triton $\mathrm{X}-100,0.1 \%$ bovine serum albumin, $0.05 \%$ sodium azide, $\mathrm{pH}$ 7.2).

Fixed and permeabilized animals were placed in block buffer $(\mathrm{AbB}+$ $5 \%$ normal goat serum) for $1 \mathrm{~h}$ at room temperature, then in block buffer plus a 1:100 dilution of mouse anti-MEC-2, mouse anti-MEC-4 or mouse anti-MEC-5 sera for $12-15 \mathrm{~h}$ at $4^{\circ} \mathrm{C}$. Labeled nematodes were washed with $\mathrm{AbB} 3$ times, 10 min each, then incubated in either goat anti-mouse or goat anti-rabbit secondary antibodies with a conjugated 568 or $488 \mathrm{~nm}$ Alexafluor, respectively (Invitrogen, Carlsbad, CA) for $2 \mathrm{~h}$ at room temperature. Unbound secondary antibody was washed off and nematodes were mounted with Prolong Antifade medium (Invitrogen) and imaged with a Zeiss (Oberkochen, Germany) Axiocam mounted on a Zeiss Axioplan 2 microscope with a Zeiss Plan-Neofluar $100 \times / 1.4$ numerical aperture (NA) oil objective. Double label experiments used the same labeling protocol as single label experiments. For display purposes only, image contrast was adjusted in Adobe Photoshop CS2, version 9.0 (Adobe Systems, San Jose, CA). Unless indicated, immunofluorescent puncta were identified on the basis of their intensity compared with background; the size and interpunctum intervals (defined as the edge-toedge distance between adjacent puncta) were measured with the ImageJ software program (Abramoff et al., 2004). To compare IF puncta with fluorescent beads and simulated IF puncta, we identified IF puncta as peaks in background-subtracted intensity profiles and recorded their length as the full-width at half-maximum (FWHM) of each peak and the interpunctum interval as the peak-to-peak distance.

Colocalization of immunofluorescent puncta. Image colocalization was quantified as described by Li et al. (2004). Paired images from double label experiments were prepared and analyzed in parallel using ImageJ. Images were cropped to include only TRNs and processed with the "subtract background" function. Next, the images were converted into 8-bit monochrome format and colocalization of overlapping puncta quantified using Intensity Correlation Analysis (ICA) in the JACoP plugin for ImageJ. The intensity correlation quotient (ICQ) is a metric of the association of pixel intensities $(-1=$ anti-correlated, $0=$ uncorrelated, $+1=$ correlated).

Serial-section immunoelectron microscopy. Adult wild-type, N2 nematodes were prepared for immunoelectron microscopy as previously described (Muller-Reichert et al., 2003; McDonald and Auer, 2006). A Leica (Vienna, Austria) AFS freeze substitution apparatus was used to preserve and embed the nematodes in $0.2 \%$ glutaraldehyde (GA) and LR-White resin, respectively. Serial, ultrathin sections $(50 \mathrm{~nm})$ were cut with a diamond knife on a Leica Ultracut $S$ microtome and collected on Formvar-coated, nickel slot grids. The grids were next floated on $50 \mu \mathrm{l}$ of block solution ( $1 \times$ PBS, $0.1 \%$ Tween $20,0.1 \%$ BSA, $0.1 \%$ cold water fish gelatin, $0.01 \%$ sodium azide, $\mathrm{pH} 8.2$ ) for $30 \mathrm{~min}$, transferred to a $50 \mu \mathrm{l}$ drop of block solution containing a 1:100 concentration of preadsorbed MEC-2, MEC-4, or MEC-5 sera for $1 \mathrm{~h}$. The grids were then washed five times on $50 \mu$ d drops containing wash solution ( $1 \times$ PBS, $0.1 \%$ Tween 20 , $\mathrm{pH}$ 8.2) for $5 \mathrm{~min}$ followed by flotation on a drop of 1:50 concentration of Goat anti-Mouse Fab' fragment conjugated to a $15 \mathrm{~nm}, 10 \mathrm{~nm}$, or $5 \mathrm{~nm}$ gold bead in block solution for 30 min (Ted Pella, Redding, CA). Next, unbound secondary antibody was removed with a salt wash $(1 \times \mathrm{PBS}$, $2.5 \mathrm{M} \mathrm{NaCl}, 0.1 \%$ Tween $20, \mathrm{pH} 8.2$ ) for $5 \mathrm{~min}$ followed by five rounds of washing with $\mathrm{ddH}_{2} \mathrm{O}$ for 5 min each. Grids were postfixed on $2 \%$ GA for $5 \mathrm{~min}$. To enhance contrast, labeled sections were poststained in 3.5\% uranyl acetate in acetone for $2 \mathrm{~s}$, and then Reynold's lead citrate preparation for $2 \mathrm{~min}$. The grids were imaged on a Jeol (Tokyo, Japan) TEM1230 transmission electron microscope and images acquired with a Gatan 967 slow-scan, cooled CCD camera (Gatan, Pleasanton, CA).

We collected $50 \mathrm{~nm}$ sections from five wild type adult C. elegans. These sections contained cross-sections of one ALM, six PLMs, one AVM and one PVM. Approximately one-third of the sections were consecutive. Three groups of consecutive sections were used to construct threedimensional (3-D) models of MEC-2 and MEC-5 distribution in the TRN: 1) $7.5 \mu \mathrm{m}$ reconstructed from 151 serial sections labeled with anti-MEC-2, including two adults, one ALM and two PLMs; 2) $20 \mu \mathrm{m}$ reconstructed from 400 serial sections labeled with anti-MEC-2, including one adult and two PLMs; 3) $1 \mu \mathrm{m}$ reconstructed from 20 serial 
sections labeled with anti-MEC-5, including one adult and two PLMs. The remaining sections were nonconsecutive.

Analysis of SS-IEM datasets. ImageJ (Abramoff et al., 2004) was used to measure the position of gold labels and ECM position in individual immunoelectron micrographs. The coordinate of each label cluster was plotted as $(r \cos \theta, r \sin \theta)$ measured in polar coordinates from individual micrographs, where $r$ was the normalized distance from the center of each TRN cross-section and $\theta$ was the angle. Neurite profiles were typically elliptical having major and minor axes of $514 \pm 132$ (mean \pm SD, $n=69$ ) and $359 \pm 84 \mathrm{~nm}$, respectively. Average ECM density was computed by measuring the angular distribution of detectable, osmophilic ECM, creating an idealized image representing that distribution and computing an average of all such idealized ECM distributions.

Three-dimensional models were generated with Reconstruct (Fiala, 2005). Briefly, all images from consecutive sections were aligned manually and the outline of all subcellular structures traced by hand. Distances between structures were measured in three dimensions using Reconstruct. Average values reported as mean \pm SD. Open-source software (Blender3D, http://www.blender3d.org) was used to generate the animations shown in supplemental data (available at www.jneurosci.org as supplemental material).

Immunofluorescence simulations. To simulate the immunofluorescent puncta that arise from our SS-IEM datasets under ideal conditions, we first computed the 2-D distribution of light intensity, $I$, produced by a point source observed through an aberration-free lens or the Airy disk: $I(r, 0)=I_{0}\left[2 J_{1}(r) / r\right]^{2}$, where $r$ is the radial distance from the optical axis, $I_{0}$ is the intensity at $r=0$ and $J_{1}$ is a first-order Bessel function (Born and Wolf, 1980). The lens we simulated matched the objective used to acquire wide-field immunofluorescent images and had an NA of 1.4. We assumed that light emitted from the point source had a wavelength of 605 $\mathrm{nm}$; the peak of the emission curve for the Alexa Fluor $568 \mathrm{~nm}$ conjugated secondary antibody. A complete simulation was constructed by placing an Airy disk at each position at which a gold bead was detected in each SS-IEM data set and computing the sum of the Airy disks. Both membrane-associated and cytoplasmic beads were included in the simulation. For the purposes of simulations, all beads were assumed to be in the same focal plane because the width of the TRN neurite is much smaller than the theoretical resolution along the optical $(\mathrm{z})$ axis ( $z$-axis resolution $\approx 935 \mathrm{~nm}$, neurite width $=512 \mathrm{~nm}$ ). We note that the simulation underestimates puncta size because it does not account for dispersion and light scattering, mismatches in refractive index of the immersion oil, glass coverslip, and the mounting media, or for chromatic dispersion.

\section{Results \\ MeT channels subunits and MEC- 5 collagen form distinct puncta in TRN neurites}

The partial loss of touch sensitivity conferred by temperaturesensitive mutations in mec-5 is enhanced by mutations in genes encoding MeT channel subunits including mec- 2 and $m e c-4$, suggesting that MEC-5 collagen interacts with MeT channel complexes and could act as a gating tether (Gu et al., 1996). Although previous reports have supported this idea (Emtage et al., 2004), both MEC-4 and MEC-5 were visualized using fluorescent fusion proteins. As such fusion proteins are likely to be over-expressed in transgenic C. elegans, we re-examined the distribution of native amounts of MEC-2, MEC-4 and MEC-5 in wild-type animals. We focused on the four lateral TRNs (ALMs and PLMs which innervate the anterior and posterior halves of the body, respectively).

All three antibodies labeled TRN cell bodies and discrete puncta along the length of TRN neurites (Fig. 1A). Little is known about how such puncta are formed or the patterning process that controls their positioning except that wild-type MEC-5 and MEC- 6 are required for their formation (Chelur et al., 2002; Emtage et al., 2004). To learn more, we determined the distribution of interpunctum intervals. In principle, if the underlying

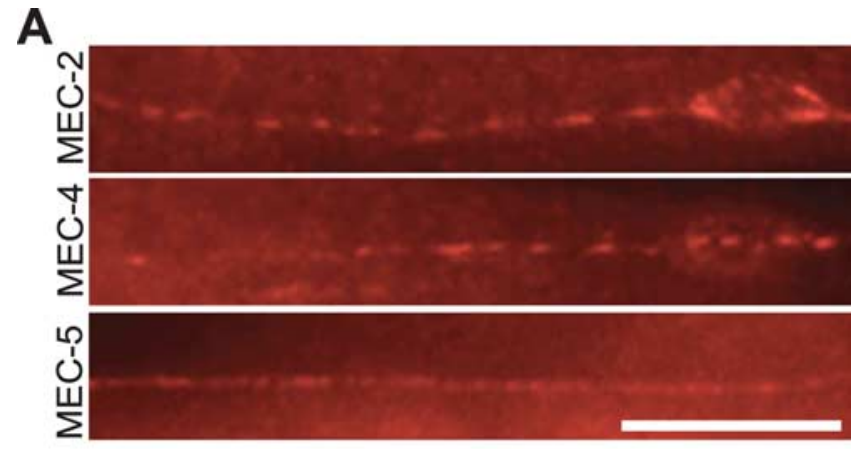

B

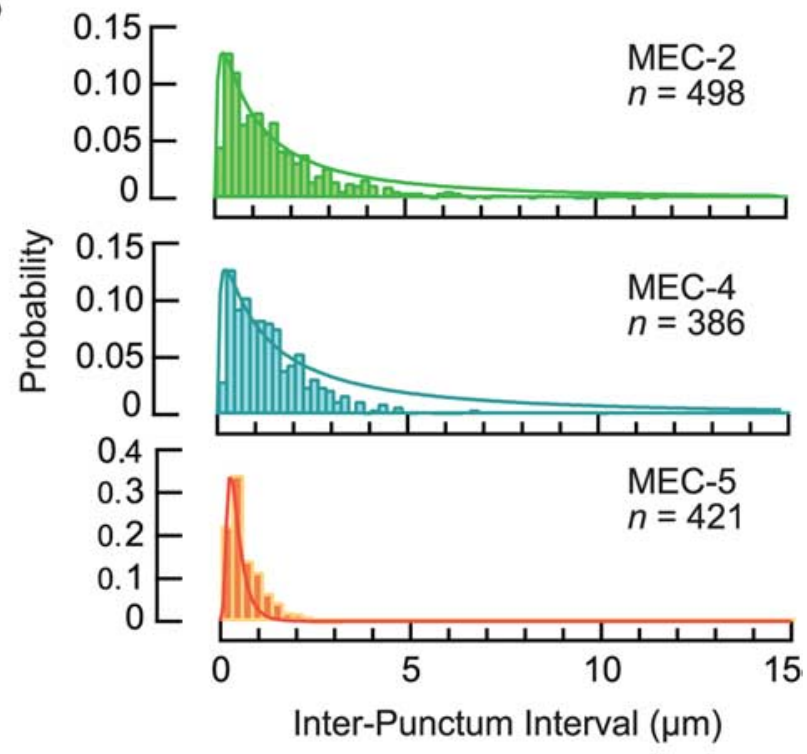

C

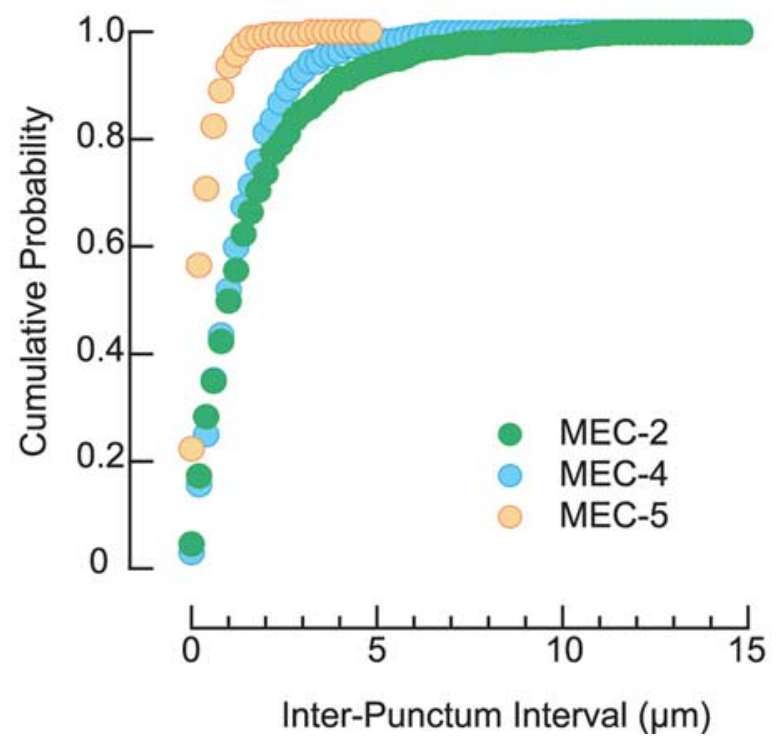

Figure 1. Native MeT channels and MEC-5 collagen decorate TRN neurites. $\boldsymbol{A}$, Indirect immunofluorescence labeling of PLM in wild-type animals. Similar results were observed in 22, 18, and 15 neurites labeled with anti-MEC -2 , anti-MEC -4 , and anti-MEC -5 antibodies, respectively. Anterior is to the left. Scale bar, $10 \mu \mathrm{m}$. B, Probability distributions (pdfs) of interpunctum intervals for MeT channel subunits (MEC -2 and MEC -4 ) and MEC -5 collagen. Smooth curves were fit to the data according to a log-normal distribution. Fitting parameters were as follows (peak, width in $\mu \mathrm{m}$ ): MEC-2 $(0.2,2.15)$, MEC-4 $(0.2,2.34)$, and MEC-5 $(0.263,0.84)$.C, Cumulative probability distribution functions for the data shown in $\boldsymbol{B}$. 
patterning process is regular and precise but puncta detection and visualization methods are subject to random noise, the distribution of intervals is expected to be normal (Gaussian). Instead, we found that the distribution of interpunctum intervals was log-normal (Fig. $1 B, C$ ), suggesting that patterning is the result of the product of two (or more) random processes (Limpert et al., 2001). MEC-2 and MEC-4 intervals had similar distributions, including a significant proportion of intervals that were longer than $2 \mu \mathrm{m}$. The distribution of MEC-5 intervals was more compact and no interval was longer than $2 \mu \mathrm{m}$. On average, MEC-2 and MEC-4 IF puncta were separated by $2.6 \pm 2.7 \mu \mathrm{m}$ ( $n=498$ intervals $)$ and $1.7 \pm 1.9 \mu \mathrm{m}(n=386$ intervals $)$, respectively, whereas MEC-5 IF puncta were separated by only $0.65 \pm$ $0.6 \mu \mathrm{m}(n=421$ intervals $)$.

Double-labeling experiments revealed that, whereas MEC-2 and MEC-4 IF puncta were colocalized (Fig. 2B), MEC-2 and MEC-5 IF puncta frequently occupied distinct domains of lateral neurites (Fig. 2C). We quantified the extent of colocalization using an intensity-based measure of the covariance of pixel intensities in the two channels (Li et al., 2004). Samples colabeled with polyclonal antibodies raised against MEC-2 in mouse (this study) and rabbit (Zhang et al., 2004) served as a control for the values expected for colocalized proteins (Fig. $2 \mathrm{~A}$ ). This analysis strongly supports the conclusion that MEC-2 colocalizes with MEC-4 but not with MEC-5 (Fig. 2D).Together, our analysis of immunofluorescent puncta implies that, in wild-type animals: (1) the MeT channel subunits (MEC-2 and MEC-4) are patterned by a mechanism that is distinct from the one that governs the patterning of the ECM protein, MEC-5 and (2) MEC-5 is unlikely to be a subunit of the sensory MeT complex in vivo.

\section{Circumferential distribution of MeT channels, MEC-5 collagen, and ECM}

We used serial-section immunoelectron microscopy (SS-IEM), to determine the in vivo localization of MeT channel complexes and MEC-5 collagen with nanometer resolution. To maximize the chances of detecting MeT channels and MEC- 5 collagen both of which are patterned on a micron-scale (Fig. $1 B, C$ ), we cut thin $(50 \mathrm{~nm})$ cross-sections and retained serial sections sufficient to reconstruct micron-scale segments of five TRN neurites. TRN neurites innervate the cuticle, are positioned within $150 \mathrm{~nm}$ of the inner surface of the cuticle and, in adult animals, they are engulfed by and embedded within the hypodermis (Fig. $3 A, B$ ). Each neurite is coated by an electron-dense material (Fig. $3 B$ ) which is believed to comprise a specialized ECM needed for touch sensation (Du et al., 1996). In general, the apical or cuticle side was more likely to be coated by this electron-dense material than was the basal side (Fig. 3B).

Anti-MEC-4 and anti-MEC-2 antibodies decorated two structures in wild-type TRNs, the plasma membrane and the $15 \mathrm{pf}$ microtubules, whereas anti-MEC-5 antibodies decorated the space in between TRN neurites and the surrounding hypodermal cell (Fig. 3C) (supplemental Fig. 1, available at www.jneurosci.org as supplemental material). Approximately half of the MeT channel labels were associated with the plasma membrane: $51 \%$ $(n=49)$ and $44 \%(n=36)$ of anti-MEC- 2 and anti-MEC-4 labels, respectively). These labels were distributed around the circumference of the TRN neurite without any obvious bias for either the apical or basal aspect of the neurite (Fig. $3 D, E$ ). In some cases, clusters of gold labels were associated with the plasma membrane. Half of the membrane-associated anti-MEC-4 labels were doublets, whereas approximately one-fifth of the membrane-associated anti-MEC-2 labels contained clusters of 2

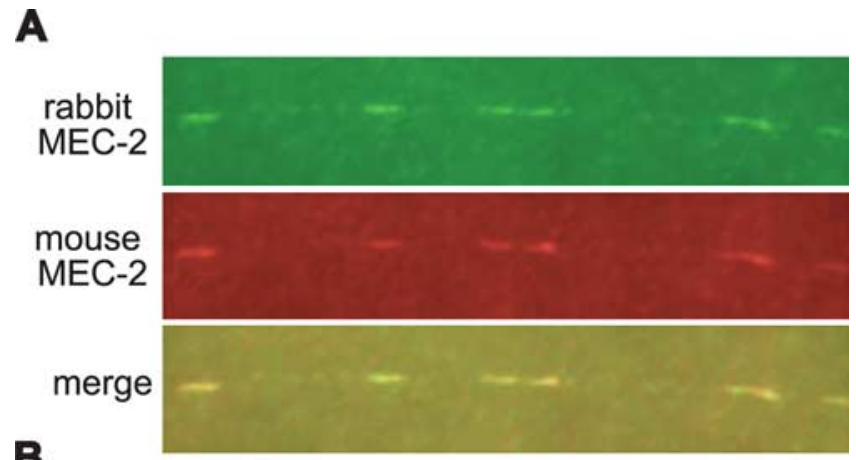

B
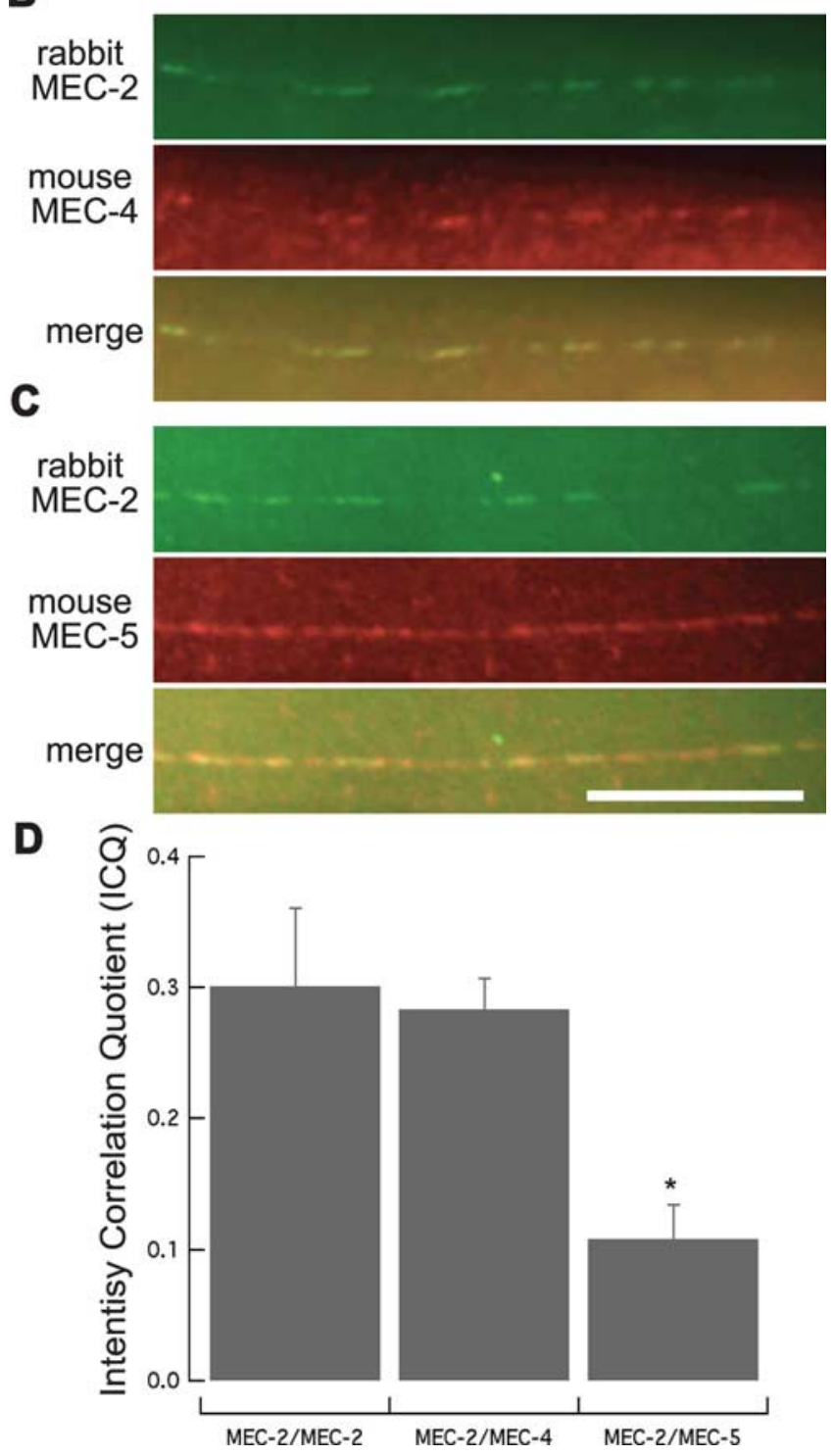

Figure 2. The MEC-2 channel subunit colocalizes with MEC-4 but not with MEC-5. Indirect immunofluorescence double labeling of TRNs with antibodies specific for the following: $\boldsymbol{A}$, two distinct domains of MEC-2; $\boldsymbol{B}$, MEC-2 and MEC-4; and $\boldsymbol{C}$, MEC-2 and MEC-5. D, Quantification of colocalization in the double-label experiments. Intensity correlation quotient values are as follows (average $\pm S D, n$ indicates double-labeled neurites): MEC-2/MEC-2 $(0.3 \pm 0.06 ; n=$ 8), MEC-2/MEC-4 (0.28 $\pm 0.02 ; n=8)$, and MEC-2/MEC-5 $(0.11 \pm 0.03 ; n=8) .{ }^{*} p<0.01$, Student's $t$ test. Scale bar, $10 \mu \mathrm{m}$.

or 3 gold beads. Anti-MEC-5 antibodies were significantly more likely to be found on the apical side of TRN neurites (Fig. 3D,E) (supplemental Video 1, available at www.jneurosci.org as supplemental material). Like our immunofluorescence findings, 
A

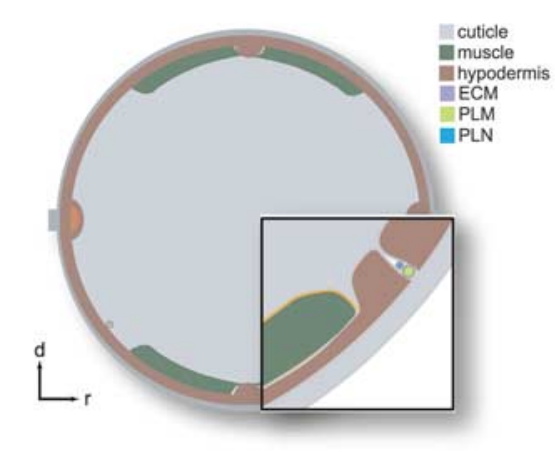

B

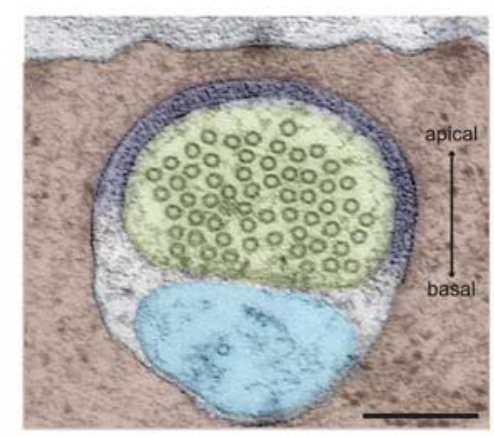

C

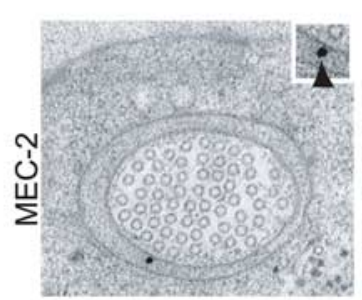

D

E
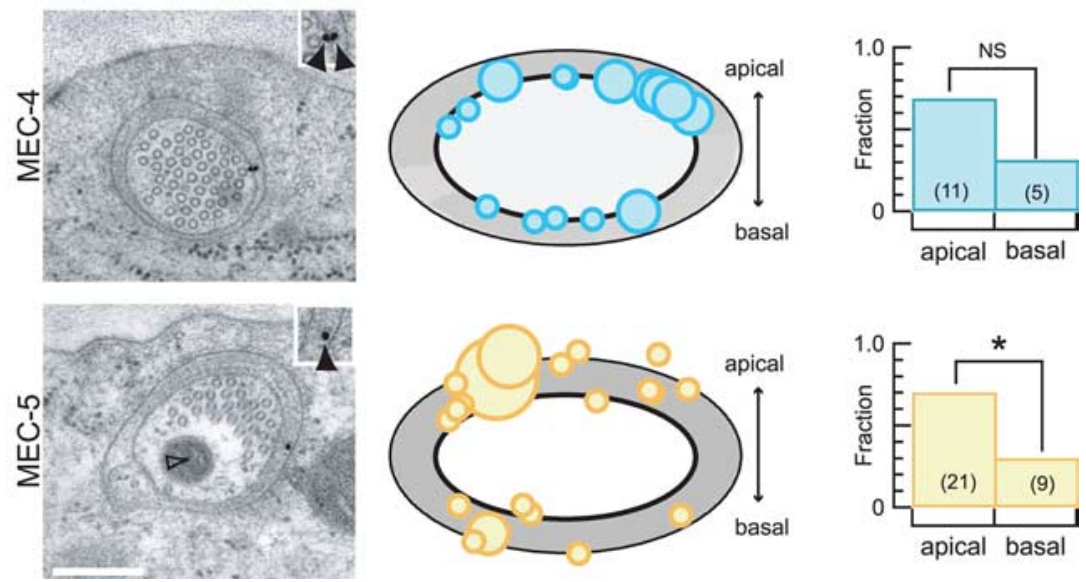

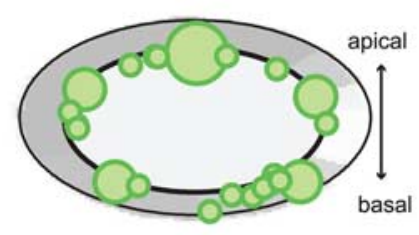

inability to detect synaptic vesicles generally, because many were detected in neurons in the ventral nerve cord (supplemental Fig. 2, available at www.jneurosci.org as supplemental material). We note, in addition, that the space between 15-pf microtubules $(\sim 10 \mathrm{~nm})$ is too small to accommodate even synaptic vesicles which are 30-35 $\mathrm{nm}$ in diameter in electron micrographs of conventionally prepared samples (White et al., 1986; Nonet et al., 1999) and $34 \pm 4 \mathrm{~nm}$ $(n=14)$ in samples prepared using HPF-FS (this study). Together, these observations suggest that few, if any vesicles are present in wild type TRN neurites in adult animals.

\section{Efficiency and specificity of serial-} section immunoelectron microscopy

As expected from the micron-scale interpunctum spacing observed using immunofluorescence (Fig. 1), gold beads were detected in a minority of thin sections labeled with either anti-MEC-4 or anti-MEC-2 antibodies ( 85 of $\sim 2500$ sections or $\sim 3 \%$ ). Labels were not present in sections flanking those bearing membrane-associated antiMEC-4 and anti-MEC-2 labels (Fig. 4), suggesting that $\mathrm{MeT}$ channels are not concentrated into extended patches. This finding is consistent with the observation that the majority of IF puncta were the same size as the image of single fluorescent beads smaller than the theoretical diffraction limit (see Fig. 6B). Consistent with the shorter interpunctum intervals of MEC-5 IF puncta (Fig. 1), gold beads were detected approximately twice as often in thin sections labeled with anti-MEC- 5 antibodies (26 of 300 or $\sim 9 \%$ ). Although anticipated from the distribution of IF puncta, the sparseness of labeling at the electron microscopic level raised concerns regarding both the efficiency and specificity of labeling in thin sections. Also, thin sections were embedded in a plastic resin (LR White) that may limit antibody penetration (Griffiths and Hoppeler, 1986; Lucocq, 1994); however, Micheva and Smith (2007) report that antibodies can penetrate LR White sections as these data argue against an obligatory physical interaction between MeT channels and MEC- 5 collagen.

The remaining MeT channel labels were associated with 15pf microtubules distributed throughout the cytoplasm (supplemental Fig. 1, available at www.jneurosci.org as supplemental material). Intracellular labels were not associated with vesicles in TRN neurites and vesicles were not detected in any of our five SS-IEM reconstructions, which covered a total length of $63 \mu \mathrm{m}$ (data not shown). The lack of vesicles in adult TRN neurites was not attributable to an inability to resolve membranes in TRNs, however, because plasma membranes and other membranebound organelles such as mitochondria were readily detected (Fig. 3C, bottom). Nor was the lack of vesicles attributable to an thick as $200 \mathrm{~nm}$. In the worst case, only those antigens on the surface of each thin section are available to be labeled and our sampling interval is equal to the thickness of each section or $50 \mathrm{~nm}$. We evaluated the impact of the $50 \mathrm{~nm}$ sampling interval by using our SSIEM datasets to simulate immunofluorescence images and by analyzing these simulated images using the same methods we used to analyze experimental immunofluorescence images. To compare experimental and simulated immunofluorescence images, we compiled histograms of interlabel intervals. Consistent with the idea that most if not all channel complexes detected by immunofluorescence were detected by SS-IEM, the cumulative distribution functions derived from these histograms were indistinguishable (see Fig. 6C).

We examined specificity in three ways. First, we reasoned that 
$-100$

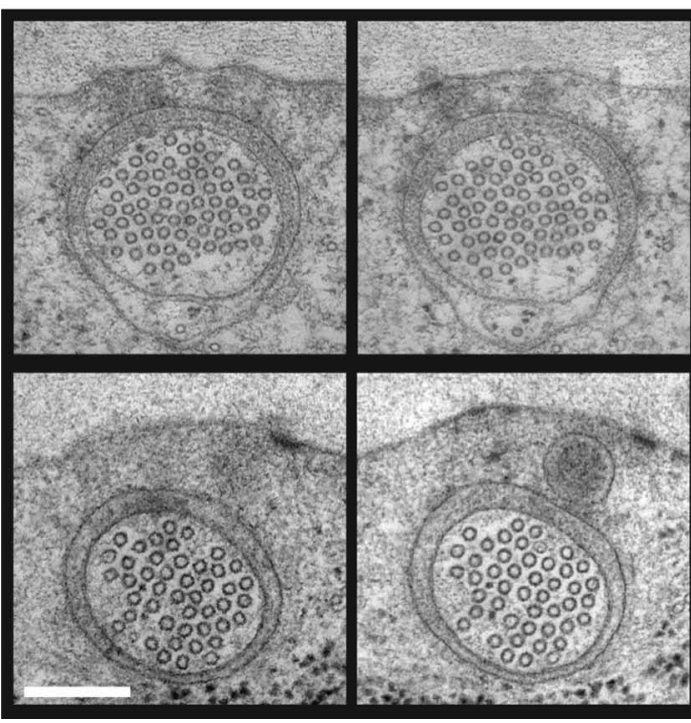

0 $+50$ $+100 \mathrm{~nm}$

Figure 4. Sequential immunoelectron micrographs of $50 \mathrm{~nm}$ sections labeled with anti-MEC-2 (top) and anti-MEC-4 (bottom). Scale bar, $200 \mathrm{~nm}$.

if the antibodies are specific for their target proteins, then they should fail to label TRNs in null mutants. Using immunofluorescence microscopy, we found that, although TRN neurites were intact and grossly normal in mec-4, mec-2 and mec-5 null mutants, they were not labeled by antibodies directed against the respective gene products (supplemental Fig. 3, available at www.jneurosci.org as supplemental material). Second, we compared the density of gold beads in TRNs with that in the surrounding hypodermal cell, which does not express mec-4 or mec- 2 and presents a much larger cross-sectional area than the TRN neurite, and found that labeling density was 8.5-fold higher in TRNs than in hypodermal cells. Third, qualitatively similar results were obtained with three different nanogold-conjugated secondary antibodies, a result which argues against the idea that the labeling pattern is attributable to nonspecific labeling by the secondary antibody.

\section{Nanoscale position of membrane-associated MeT channels and $15 \mathrm{pf}$ microtubules}

TRN neurites are filled with a cross-linked bundle of microtubules. Individual microtubules are 10-20 $\mu \mathrm{m}$ long on average and are staggered throughout the entire length of each TRN neurite (Chalfie and Thomson, 1979). We identified distal endpoints of $15 \mathrm{pf}$ microtubules by virtue of the absence of a microtubule cross-section in the subsequent (more distal) section. Distal endpoints were more likely to occur at the periphery of the microtubule bundle than at its center (Fig. $5 A, B$ ). Approximately half (134 of 270) of the distal endpoints appeared to make direct contact with the plasma membrane; such contact sites were characterized by a diffuse patch of electron-dense filamentous material and present at a longitudinal frequency of $4.1 \pm 1 \mu \mathrm{m}^{-1}(n=$ 5 TRN segments). It has been suggested that MeT channels localize to these junctions ( $\mathrm{Gu}$ et al., 1996). As noted previously (Emtage et al., 2004), this seems unlikely because the average longitudinal frequency of distal endpoints exceeds that of MEC-2 and MEC-4 IF puncta by 10-fold. [The average frequencies of MEC-2 and MEC-4 IF puncta are $0.3 \pm 0.1 \mu \mathrm{m}^{-1}$ ( $n=22$ neurites) and $0.38 \pm 0.07 \mu \mathrm{m}^{-1}(n=18$ neurites $)$, respectively.]
To learn more about the relationship between membraneassociated MeT channels and distal endpoints, we reconstructed segments of five TRNs (three $7.5 \mu \mathrm{m}$ segments and two $20 \mu \mathrm{m}$ segments) from SS-IEM data sets labeled with anti-MEC-2 antibodies and generated three-dimensional digital renderings of the following structures: TRN plasma membrane, $15 \mathrm{pf}$ microtubules, anti-MEC-2 labels, cuticle, and the PLN plasma membrane. One of these models is shown in Figure $5 D$; for clarity only the plasma membrane, anti-MEC-2 labels and terminating microtubules are shown (all microtubules were reconstructed, however). An animated "fly-through," which demonstrates the spatial relationship between membrane-associated channels and microtubule endpoints, is provided in supplemental data (supplemental Video 2, available at www.jneurosci.org as supplemental material). We found a total of 17 membraneassociated channels in the five reconstructed TRN segments. Three labels were $>1 \mu \mathrm{m}$ away from the nearest microtubule endpoint and were not analyzed further. Of the remaining 14 channels, none were closer than $141 \mathrm{~nm}$ to the nearest distal endpoint (Fig. $5 E$ ). On average, $346 \pm 178 \mathrm{~nm}(n=14) \mathrm{sep}-$ arated $\mathrm{MeT}$ channels from the nearest 15pf microtubule distal endpoint. These data indicate that membrane-associated MeT channels are neither localized to junctions between microtubule endpoints and the plasma membrane nor likely to interact directly with such endpoints. Additionally, there was no obvious geometric relationship between membraneassociated MeT channels and en passant microtubules, suggesting that interactions between MeT channels and $15 \mathrm{pf}$ microtubules are likely to be indirect.

\section{Evidence that the 15pf microtubule bundle is a structural organelle}

Previously we showed that, although native MeT channels can be activated in mec-7 mutant TRNs lacking 15pf microtubules, the pressure required to open half of the channels is increased twofold in mec-7 mutants (O'Hagan et al., 2005). To learn more about how 15pf microtubules might contribute to mechanotransduction, we characterized the cytoskeletal structures con- 
A

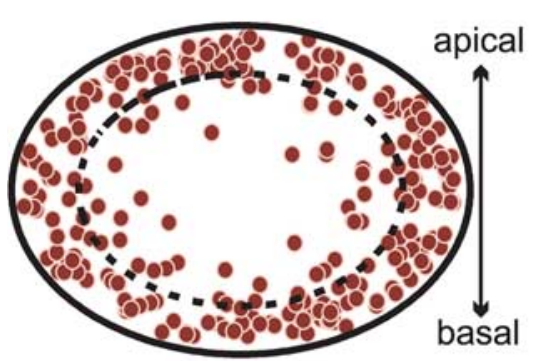

B

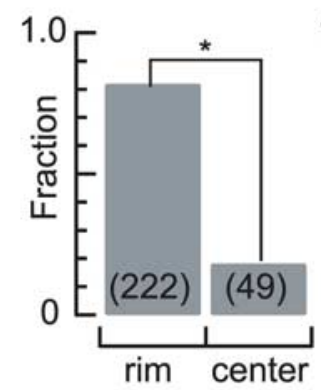

D

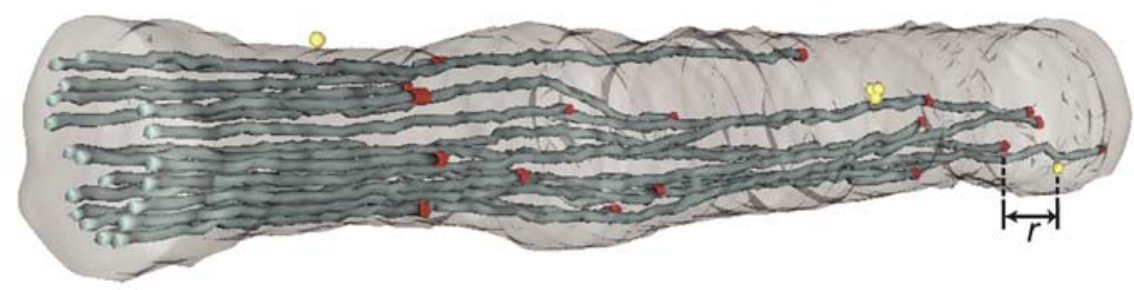

E

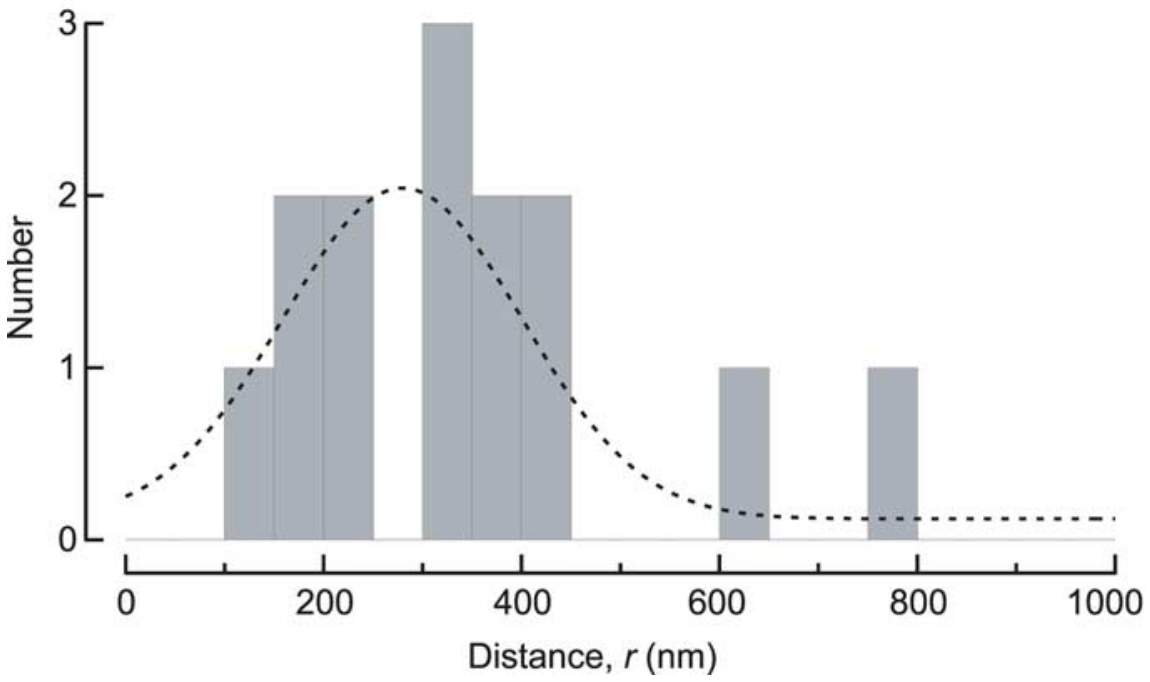

Figure 5. Position of distal endpoints of $15 \mathrm{pf} \mathrm{microtubules} \mathrm{and} \mathrm{the} \mathrm{distance} \mathrm{between} \mathrm{endpoints} \mathrm{and} \mathrm{native} \mathrm{MeT} \mathrm{channels.} \boldsymbol{A}$, Position of distal endpoints detected in SS-IEM reconstructions of lateral TRN neurites. Dashed line indicates the border between the center and rim of a normalized TRN profile; the center and rim zones have equal areas. $\boldsymbol{B}$, Fraction of endpoints in the rim and center of the TRN cross-section. ${ }^{*} p<0.0001, \chi^{2}$ test. $C$, Fraction of endpoints on the apical and basal sides of the TRN. Numbers in each category indicated in parentheses. $D, 3-D$ digital model of a $7.5 \mu \mathrm{m}$ fragment of PLM derived from 151 serial sections labeled with anti-MEC-2 antibody. For clarity, only the following structures are shown: the plasma membrane (transparent gray); terminating microtubules (aqua) and their distal endpoints (red); and plasma membrane-associated MeT channels (gold spheres). An animated fly-through of the model is included in supplemental data (available at www.jneurosci.org as supplemental material). The distance between each membrane-associated channel and the nearest microtubule endpoint was measured as illustrated. $\boldsymbol{E}$, Histogram of the distance between plasma membrane-associated MeT channels and the nearest microtubule endpoint. The average and median distances were 347 and $328 \mathrm{~nm}$, respectively. Measurements were made of 271 distal $15 \mathrm{pf}$ microtubule endpoints and endpoint-to-channel distances for 14 channels localized in five 3-D models derived from SS-IEM of lateral TRNs (4 PLMs and 1 ALM).

necting all $15 \mathrm{pf}$ microtubules into a bundle and the network of links attaching the entire $15 \mathrm{pf}$ microtubule bundle to the plasma membrane (see Fig. 7). Inter-microtubule links, which were more numerous than previously described (Chalfie and Thomson, 1979, 1982), were $9.7 \pm 2.2 \mathrm{~nm}$ in length (Fig. 7C). Collectively, they attached all of the microtubules within the bundle to

C

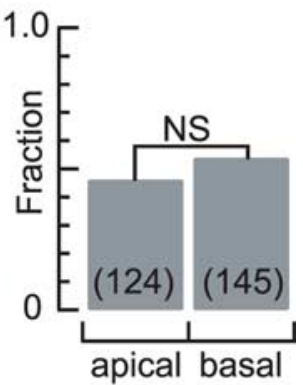

each other, suggesting that, like axonemal microtubules, TRN microtubules are constrained to move together as a single structure. In a typical $50 \mathrm{~nm}$ section, central microtubules were surrounded by an average of $6 \pm 1$ microtubules (90 central microtubules from 4 sections), whereas those on the periphery of the bundle were surrounded by only $4 \pm 1$ microtubules (71 peripheral microtubules from 4 sections). Furthermore, an average of $5 \pm 2$ links radiated from the surface of each 15pf microtubule (194 microtubules from 5 TRN sections), suggesting that the links hold the microtubules in a hexagonal array. The outward facing side of each peripheral microtubule radiated an average of $3 \pm 1$ links to a network of structurally distinct filaments that connect the microtubule bundle to the plasma membrane (Fig. 7).

The filaments linking the microtubule bundle to the plasma membrane were organized into a lattice of polygons. The filaments extended an average of $13.7 \pm 3.6$ nm (Fig. 7D) before changing their orientation $92 \pm 26^{\circ}$ (113 angles from $10 \mathrm{sec}$ tions). The lattice of filament-like structures filled the cytoplasmic space between the microtubule bundle and plasma membrane. Individual filaments contacted all regions of the inner leaflet of the plasma membrane, suggesting that the microtubule bundle is attached to the plasma membrane isotropically. We estimated that an average of 367.4 filaments contacted the inner leaflet of the plasma membrane in each $50 \mathrm{~nm}$ section by dividing the average size of the TRN plasma membrane (TRN perimeter $1.396 \pm 0.337 \mu \mathrm{m}, 50$ TRN crosssections) by the average distance between pairs of membrane attached filaments $(3.8 \pm 3.5 \mathrm{~nm}, 118$ filament pairs from 4 sections). Occasionally, a dense aggregation of filament structures contacting the plasma membrane was observed (data not shown). In these instances, individual filaments overlapped and were too numerous to count accurately.

Next, we asked whether or not such filaments could be attached to MeT channels and serve as gating tethers in place of $15 \mathrm{pf}$ microtubules. This idea makes two predictions: (1) most, if not all filaments would be positioned within nanometers of a membrane-associated MeT channel; (2) all membrane-associated MeT channels would be contacted by at least one filament. In this scenario, MeT channels could be activated by tether displacement and the lattice of filaments and bundle of 15pf microtubules would act as a mechanical amplifier. However, many $(32 \%, n=41)$ membrane-associated MeT channel labels were $>5 \mathrm{~nm}$ away from one of these filaments. This finding indicates that if such filaments are gating tethers, then they are present in 
vast excess compared with membraneassociated MeT channels and that approximately one-third of membrane-associated MeT channels are not available to be activated.

\section{Discussion}

Previous models of sensory mechanotransduction by $C$. elegans touch receptor neurons proposed a trans-cellular complex in which displacement of the ECM (or 15pf microtubules), resisted by the $15 \mathrm{pf}$ microtubules (or ECM), gates the channel in the plasma membrane (Gu et al., 1996). This model and derivatives that propose that channels are linked to the ECM but not to 15 pf microtubules (Emtage et al., 2004; Bounoutas and Chalfie, 2007) make specific predictions about the position of $\mathrm{MeT}$ channels in relation to MEC-5 and $15 \mathrm{pf}$ microtubule endpoints. We used serialsection immunoelectron microscopy and digital 3-D reconstruction methods to localize native MeT channels in C. elegans touch receptor neurons to establish the structural correlates of channel gating and to test these models. Serial-section immunoelectron microscopy provides a means for testing whether or not genetic interactions are likely to be mediated by physical association in their native environment. Because the rarity of MeT channels limits the use of traditional biochemical tests of physical interaction, this approach is especially critical for analysis of the cellular machinery responsible for sensory mechanotransduction.

Several lines of evidence support the conclusion that MeT channels, MEC-5 collagen and 15pf microtubules do not assemble into a common trans-cellular apparatus responsible for detecting touch in C. elegans TRNs. First, in wild-type animals expressing native levels of all three proteins, MEC- 2 colocalizes with MEC-4, but not MEC-5 in immunofluorescent puncta. Second, whereas membrane-associated MeT channel subunits MEC-2 and MEC-4 are distributed essentially evenly around the circumference of TRN neurites, MEC-5 is concentrated principally on the apical side. Considered together, these data argue against the idea that MeT channels are physically linked to MEC-5. Third, all membrane-associated MeT channel subunits analyzed by SS-IEM were $>100 \mathrm{~nm}$ away from the nearest distal microtubule endpoint. Finally, the average longitudinal frequency of MeT channel IF puncta (0.3-0.4 per $\mu \mathrm{m})$ differs from that of microtubule endpoints $(\sim 4$ per $\mu \mathrm{m})$ and that of MEC-5 IF puncta $(\sim 1$ per $\mu \mathrm{m})$ by 10 - and 4 -fold respectively.

Genetic interaction analysis, in which two mutations have a combined effect not exhibited by either mutation alone, has been critical for establishing functional connections between genes that mutate to produce a touch-insensitive ( $\mathrm{Mec}$ ) phenotype in C. elegans. Such interactions could arise from direct physical associations between gene products, independent contributions to a common cellular function, or both. It is impossible to distinguish among these possibilities using genetic methods alone. Our structural analysis implies that interactions between temperature-sensitive alleles of $m e c-5$ and the genes encoding the four subunits of MeT channels ( mec-4, mec-10, mec-2 and mec-6) as well as similar interactions between temperature-sensitive alleles of mec-4 and mec-5 (Huang and Chalfie, 1994; Huang et al.,
1995; Gu et al., 1996) do not arise from physical interactions between MEC-5 and MeT channel complexes in adult animals. MEC-5 could act to establish and maintain MeT channel complexes earlier in development, however. In support of this idea, temperature-shift experiments show that mec-5 is needed during the L2 and L3 larval stages (Chalfie and Sulston, 1981). Our data likewise argue against the idea that genetic interactions between MEC-5 collagen and MEC-7 $\beta$ tubulin (Gu et al., 1996) reflect their incorporation into a common complex incorporating MEC-5, the MeT channels, and 15pf microtubules. Finally, although our data demonstrate that genetic interactions between MEC-2 and MEC-7 $\beta$ tubulin (Huang et al., 1995; Gu et al., 1996) are not the result of physical association at the plasma membrane, the labeling of microtubules by anti-MEC-2 (supplemental Fig. 1, available at www.jneurosci.org as supplemental material) suggests that a physical interaction may occur in the TRN cytoplasm.

Previously, we showed that both MEC-4 and MEC-10 are pore-forming subunits of the native MeT channel (O'Hagan et al., 2005). How many of each assembles to build a functional channel? At least two MEC-4 proteins appear to be required, because degeneration caused by gain-of-function mutations in mec-4, although dominant, can be suppressed in trans by loss-offunction alleles of mec-4 (Hong and Driscoll, 1994). Our structural data, which reveal that membrane-associated MEC-4 labels are either single labels or doublets of adjacent labels (no MEC-4 triplets were observed), are consistent with this genetic inference and suggest that exactly two MEC-4 proteins form each channel. Based on a recent $1.9 \AA$-resolution structure, DEG/ENaC channels appear to be trimers (Jasti et al., 2007). Thus, the simplest model that is consistent with the available data is that native $C$. elegans MeT channels are composed of two MEC-4 proteins and one MEC-10 protein.

Based on our SS-IEM datasets, which show that membraneassociated labels are separated by an average of $2.4 \mu \mathrm{m}$, we estimate that an adult TRN neurite contains on the order of 200 membrane-associated MeT channels. This figure is likely to be an 
A
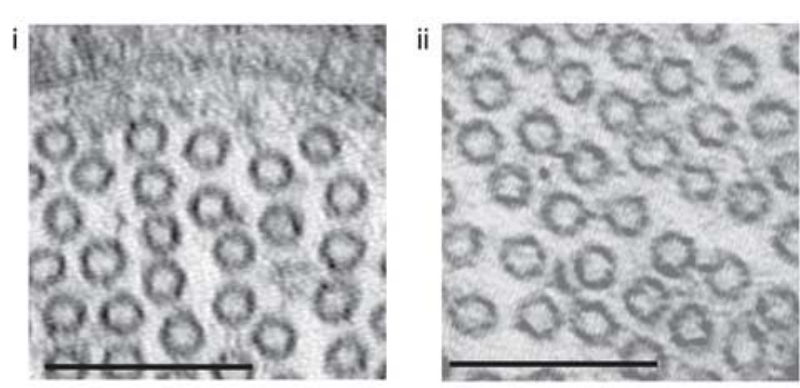

B
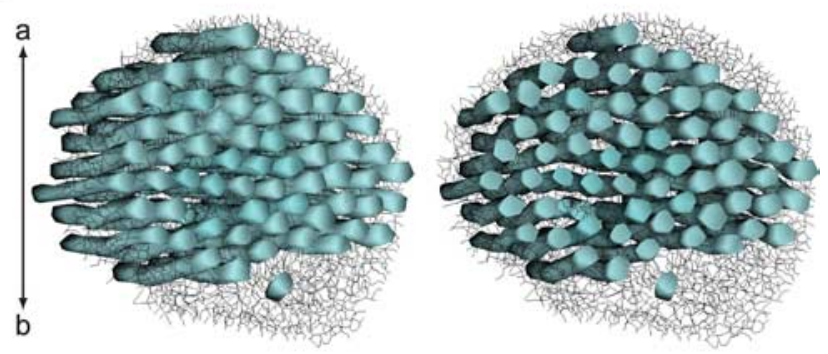

C

D
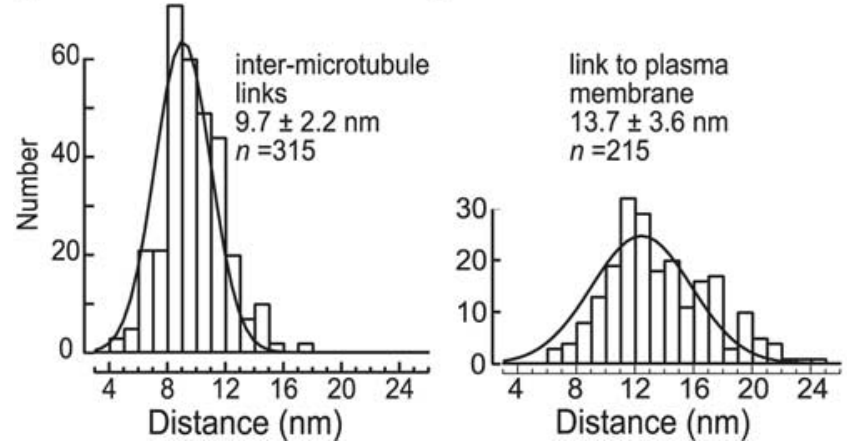

Figure 7. A network of filaments links individual 15 protofilament microtubules to each other and connects the microtubule bundle to the plasma membrane. $\boldsymbol{A}$, Electron micrographs showing filaments that link $15 \mathrm{pf}$ microtubules to one another and to the plasma membrane. Individual microtubules are linked to the majority of their nearest neighbors in the bundle. Filaments linking microtubules to the plasma membrane are more numerous and are organized into a more elaborate network. Scale bars, $150 \mathrm{~nm}$. $\boldsymbol{B}$, Digital rendering of the filaments and microtubules in four serial sections of PLM reveals the density of filaments and the extent of the connections they make. Microtubule fragments are shown in aqua, and filaments are in black. The panels are rotamers of a single 3-D digital model. $\boldsymbol{C}$, Distribution of the lengths of intermicrotubules links. A total of 315 links were analyzed in five electron micrographs of a series of consecutive $50 \mathrm{~nm}$ sections containing $6415 \mathrm{pf}$ microtubules. D. Distribution of the lengths of filaments linking the microtubule bundle to the plasma membrane. A total of 215 filaments were analyzed in the same electron micrographs as in $\boldsymbol{C}$.

overestimate, however, because a disproportionate number of interlabel intervals are longer than the average. In any case, only a fraction of these channels are activated when point loads are applied to the cuticle, because saturating mechanical stimuli activate 14-25 channels (O'Hagan et al., 2005). This implies that point loads affect only those channels present in the TRN segment directly displaced by the stimulus probe. In support of the idea that transduction is local, we showed previously that mechanoreceptor current amplitude depends not only on force, but also on the force per unit area (pressure) (O’Hagan et al., 2005). In other words, a given force activates a larger fraction of $\mathrm{MeT}$ channels when it is delivered to a smaller area.

By virtue of their material properties or thickness relative to channels or both factors, bilayers can modulate channel gating by altering the relative stability of closed and open states (McIntosh and Simon, 2006). We speculate that forces delivered via the lipid bilayer facilitate MeT channel gating in vivo. A critical role for lipids in C. elegans MeT channels is suggested by the finding that MEC-2 binds cholesterol and that at least one mutation that disrupts touch sensation also disrupts cholesterol binding (Huber et al., 2006). Additional support comes from the finding that UNC24 , which has a lipid-transfer domain and is implicated in nonvesicular transfer of lipids (Barnes et al., 1996), colocalizes with MEC-2 in vivo, coimmunoprecipitates with MEC-2 in heterologous cells, and modulates channel activity in Xenopus oocytes (Zhang et al., 2004). For this model to be viable, it is necessary to postulate a mechanism by which pressure applied to the cuticle is converted into membrane stretch. We propose that the $15 \mathrm{pf} \mathrm{mi-}$ crotubule bundle serves this function and can be viewed as a force-transmitting organelle that converts point loads applied perpendicular to TRN neurites into membrane bending or stretch via the lattice of filaments that radiate from all microtubules in the periphery of the bundle and contact the plasma membrane. Additionally, the microtubule-to-microtubule cross-links could contribute to amplification by increasing the stiffness of the microtubule bundle.

The contribution of the $15 \mathrm{pf}$ microtubules to mechanotransduction is likely to be indirect. Here, a useful analogy can be drawn to the outer pillar cells of the mammalian cochlea, which play a critical role in transferring force and displacement from the basilar membrane to the reticular lamina and the mechanosensory hair cells. Their contribution to cochlear mechanics is thought to rely on the stiffness conferred by thousands of crosslinked 15pf microtubules (Tolomeo and Holley, 1997). Mutations that alter the number of outer pillar cells and leave hair cells intact, cause deafness in mice (Shim et al., 2005; Hayashi et al., 2007), underscoring their permissive, but critical role in mammalian mechanotransduction. The present work serves a first step toward the generation of structurally and mechanically realistic models of how force and displacement applied to the skin are delivered to sensory $\mathrm{MeT}$ in $C$. elegans touch receptor neurons.

\section{References}

Abramoff MD, Magelhaes PJ, Ram SJ (2004) Image processing with ImageJ. Biophotonics Int 11:36-42.

Altun ZF, Hall DH (2005) Handbook of C. elegans anatomy. In: Worm atlas. E-book available at http://www.wormatlas.org/handbook/contents.htm.

Barnes TM, Jin Y, Horvitz HR, Ruvkun G, Hekimi S (1996) The Caenorhabditis elegans behavioral gene unc-24 encodes a novel bipartite protein similar to both erythrocyte band 7.2 (stomatin) and nonspecific lipid transfer protein. J Neurochem 67:46-57.

Born M, Wolf E (1980) Principles of optics. Oxford: Pergamon.

Bounoutas A, Chalfie M (2007) Touch sensitivity in Caenorhabditis elegans. Pflügers Arch 454:691-702.

Brenner S (1974) The genetics of Caenorhabditis elegans. Genetics 77:71-94. Chalfie M, Sulston J (1981) Developmental genetics of the mechanosensory neurons of Caenorhabditis elegans. Dev Biol 82:358-370.

Chalfie M, Thomson JN (1979) Organization of neuronal microtubules in the nematode Caenorhabditis elegans. J Cell Biol 82:278-289.

Chalfie M, Thomson JN (1982) Structural and functional diversity in the neuronal microtubules of Caenorhabditis elegans. J Cell Biol 93:15-23.

Chalfie M, Sulston JE, White JG, Southgate E, Thomson JN, Brenner S (1985) The neural circuit for touch sensitivity in Caenorhabditis elegans. J Neurosci 5:956-964.

Chambers RS, Johnston SA (2003) High-level generation of polyclonal antibodies by genetic immunization. Nat Biotechnol 21:1088-1092.

Chelur DS, Ernstrom GG, Goodman MB, Yao CA, Chen L, R O'Hagan, Chalfie M (2002) The mechanosensory protein MEC-6 is a subunit of the C. elegans touch-cell degenerin channel. Nature 420:669-673.

Corey DP, Hudspeth AJ (1979) Response latency of vertebrate hair cells. Biophys J 26:499-506. 
Du H, Gu G, William CM, Chalfie M (1996) Extracellular proteins needed for C. elegans mechanosensation. Neuron 16:183-194.

Emtage L, Gu G, Hartwieg E, Chalfie M (2004) Extracellular proteins organize the mechanosensory channel complex in C. elegans touch receptor neurons. Neuron 44:795-807.

Ernstrom GG, Chalfie M (2002) Genetics of sensory mechanotransduction. Annu Rev Genet 36:411-453.

Fiala JC (2005) Reconstruct: a free editor for serial section microscopy. J Microsc 218:52-61.

Goodman MB, Schwarz EM (2003) Transducing touch in Caenorhabditis elegans. Annu Rev Physiol 65:429-452.

Goodman MB, Ernstrom GG, Chelur DS, O’Hagan R, Yao CA, Chalfie M (2002) MEC-2 regulates C. elegans DEG/ENaC channels needed for mechanosensation. Nature 415:1039-1042.

Griffiths G, Hoppeler H (1986) Quantitation in immunocytochemistry: correlation of immunogold labeling to absolute number of membrane antigens. J Histochem Cytochem 34:1389-1398.

Gu G, Caldwell GA, Chalfie M (1996) Genetic interactions affecting touch sensitivity in Caenorhabditis elegans. Proc Natl Acad Sci USA 93:6577-6582.

Hayashi T, Cunningham D, Bermingham-McDonogh O (2007) Loss of Fgfr3 leads to excess hair cell development in the mouse organ of Corti. Dev Dyn 236:525-533.

Hong K, Driscoll M (1994) A transmembrane domain of the putative channel subunit MEC-4 influences mechanotransduction and neurodegeneration in C. elegans. Nature 367:470-473.

Huang M, Chalfie M (1994) Gene interactions affecting mechanosensory transduction in Caenorhabditis elegans. Nature 367:467-470.

Huang M, Gu G, Ferguson EL, Chalfie M (1995) A stomatin-like protein necessary for mechanosensation in C. elegans. Nature 378:292-295.

Huber TB, Schermer B, Muller RU, Hohne M, Bartram M, Calixto A, Hagmann H, Reinhardt C, Koos F, Kunzelmann K, Shirokova E, Krautwurst D, Harteneck C, Simons M, Pavenstadt H, Kerjaschki D, Thiele C, Walz G, Chalfie M, Benzing T (2006) Podocin and MEC-2 bind cholesterol to regulate the activity of associated ion channels. Proc Natl Acad Sci USA 103:17079-17086.

Jasti J, Furukawa H, Gonzales EB, Gouaux E (2007) Structure of acidsensing ion channel 1 at $1.9 \AA$ resolution and low pH. Nature 449:316-323.

Li Q, Lau A, Morris TJ, Guo L, Fordyce CB, Stanley EF (2004) A syntaxin 1, $\mathrm{G} \alpha_{\mathrm{o}}$, and $\mathrm{N}$-type calcium channel complex at a presynaptic nerve terminal: analysis by quantitative immunocolocalization. J Neurosci 24:4070-4081.
Limpert E, Stahel WA, Abbt M (2001) Log-normal distributions across the sciences: keys and clues. Bioscience 51:341-352.

Lucocq J (1994) Quantitation of gold labelling and antigens in immunolabelled ultrathin sections. J Anat 184:1-13.

McDonald KL, Auer M (2006) High-pressure freezing, cellular tomography, and structural cell biology. Biotechniques 41:137, 139, 141 passim.

McIntosh TJ, Simon SA (2006) Roles of bilayer material properties in function and distribution of membrane proteins. Annu Rev Biophys Biomol Struct 35:177-198.

Micheva KD, Smith SJ (2007) Array tomography: a new tool for imaging the molecular architecture and ultrastructure of neural circuits. Neuron 55: 25-36.

Muller-Reichert T, Hohenberg H, O’Toole ET, McDonald K (2003) Cryoimmobilization and three-dimensional visualization of C. elegans ultrastructure. J Microsc 212:71-80.

Nonet ML, Holgado AM, Brewer F, Serpe CJ, Norbeck BA, Holleran J, Wei L, Hartwieg E, Jorgensen EM, Alfonso A (1999) UNC-11, a Caenorhabditis elegans AP180 homologue, regulates the size and protein composition of synaptic vesicles. Mol Biol Cell 10:2343-2360.

O'Hagan R, Chalfie M, Goodman MB (2005) The MEC-4 DEG/ENaC channel of Caenorhabditis elegans touch receptor neurons transduces mechanical signals. Nat Neurosci 8:43-50.

Shim K, Minowada G, Coling DE, Martin GR (2005) Sprouty2, a mouse deafness gene, regulates cell fate decisions in the auditory sensory epithelium by antagonizing FGF signaling. Dev Cell 8:553-564.

Suzuki H, Kerr R, Bianchi L, Frokjaer-Jensen C, Slone D, Xue J, Gerstbrein B, Driscoll M, Schafer WR (2003) In vivo imaging of C. elegans mechanosensory neurons demonstrates a specific role for the MEC-4 channel in the process of gentle touch sensation. Neuron 39:1005-1017.

Tang DC, DeVit M, Johnston SA (1992) Genetic immunization is a simple method for eliciting an immune response. Nature 356:152-154.

Tolomeo JA, Holley MC (1997) Mechanics of microtubule bundles in pillar cells from the inner ear. Biophys J 73:2241-2247.

Walker RG, Willingham AT, Zuker CS (2000) A Drosophila mechanosensory transduction channel. Science 287:2229-2234.

White JG, Southgate E, Thomson JN, Brenner S (1986) The structure of the nervous system of the nematode Caenorhabditis elegans. Philos Trans R Soc Lond B Biol Sci 314:327-348.

Zhang S, Arnadottir J, Keller C, Caldwell GA, Yao CA, Chalfie M (2004) MEC-2 is recruited to the putative mechanosensory complex in C. elegans touch receptor neurons through its stomatin-like domain. Curr Biol 14: $1888-1896$. 